\title{
Sequence Analysis of HSP70 Gene of Leishmania major and Leishmania tropica in Chabahar and Mashhad
}

Mansour Dabirzadeh (phD)

Department of Parasitology and Mycology, Faculty of Medicine, Zabol University of Medical Sciences, Zabol, Iran

Abbas Pashaie Neghadeh (MSc)

Department of Parasitology and Mycology, Faculty of Medicine, Zabol University of

Medical Sciences, Zabol, Iran

Tahereh Davoodi (MSc)

Department of Parasitology and Mycology, Faculty of Medicine, Zabol University of

Medical Sciences, Zabol, Iran

Mohammad Hashemi(MSc)

Department of Parasitology and Mycology, Faculty of Medicine, Zabol University of Medical Sciences, Zabol, Iran

Corresponding Author: Abbas Pashaie Neghadeh

Tel: +989132533173

Email: abbaspashaie41@gmail.com

Address: Zabol University of Medical Sciences, Zabol, Iran

Received : 14 Jun 2014

Revised: 12 May 2015

Accepted: 13 May 2015

\section{ABSTRACT}

Background and Objective: Cutaneous leishmaniasis is a parasitic disease and a health problem in different parts of Iran, especially two cities of Mashhad and Chabahar. Due to morphological similarities of most Leishmania species and difference in reservoirs of $L$. major and $L$. tropica, it is necessary to determine the parasite specie to combat the disease. Thus, this study used gene sequencing and genotyping of 70-kla heat shock protein (HSP70) to differentiate the two species of Leishmania.

Methods: In this descriptive-analytical study, microscope slides and cultures were prepared from 43 patients suspected of cutaneous leishmaniasis in Chabahar and Mashhad. PCR was performed after genomic DNA extraction and then PCR products were sequenced and analyzed.

Results: 0 the 43 patients studied, 32 direct smear and culture (74.4\%) were positive and 11 (25.6\%) showed negative results, and were therefore excluded from the study. Using HSP70-specific primers, 1962 bp and 1152bp bands were observed for HSP70 of $L$. major in Chabahar and $L$. tropica in Mashhad, respectively. Based on the results, there were 18 nucleotide differences between HSP70 of $L$. major in Chabahar and $L$. tropica in Mashhad.

Conclusion: Due to the morphological similarities between Leishmania species and inability to differentiate species through parasitological methods, the HSP70 gene can be used for identification of the species, and prevention and treatment of the disease.

Keywords: HSP70 Gene, Leishmania major, Leishmania tropica, Chabahar, Mashhad. 


\section{INTRODUCTION}

Leishmaniasis is a common disease in many parts of the world, especially in the warm and tropical regions. The geographical distribution of this disease depends on its vectors (sandflies) dispersion. The uncontrolled exploitation of timber resources, mining, construction of dams, expansion of agricultural lands, creation of new irrigation methods, development of roads in forests, urbanization, increased poverty and malnutrition are considered as the factors involved in increased incidence of this disease (1). Most infections are found in wild animals such as rodents and dogs with a higher prevalence in forests or rural areas (2).

Currently, about 350 million people in 88 countries across five continents of Africa, Asia, Europe, North America and South America are at risk of leishmaniasis. Twelve million people worldwide are infected with leishmaniasis and this number includes individuals with and without apparent clinical symptoms.

Cutaneous leishmaniasis is another common form of the disease that affects 1.5 million people worldwide. More than $90 \%$ of new cases of cutaneous leishmaniasis are found in Afghanistan, Algeria, Iran, Iraq, Saudi Arabia, Syria, Brazil and Peru. The geographical distribution of leishmaniasis is limited to dispersion of sandflies, its susceptibility to cold climates, and tendency to specific blood feeding on humans and animals and internal evolution capability of the Leishmania species (2). In urban leishmaniasis (dry sore), humans and dogs are considered as the secondary or accidental reservoir hosts. Phlebotomus sergenti sandfly is the main vector of this disease in Iran, which is abundant in foothills and old urban textures. The main hotspots of the disease include Tehran, Shiraz, Mashhad, Sabzevar, Nishabur, Kerman, Bam and Saveh (3). The desert rats or rodents (mostly Gerbillinae subfamily) are the main reservoir of rural leishmaniasis (wet sore). There are three main hotspots of leishmaniasis in Iran including central and northeast hotspot, southwest and south hotspot and southeast hotspot. (4). Chabahar and Kenarak as well as Mirjaveh are the main disease hotspots in the southeast that was investigated in this study. The main reservoir in these hotspots is a desert rat named Meriones hurrianae, which is more abundant in the western part of the Indian subcontinent. Tatra Indica is likely to act as the secondary animal reservoir in this area. While $P$. papatasi is the vector of the disease, other Phlebotomus species such as P. Salehi may also play a role in the transmission of the disease (4). Due to the differences between the reservoirs of two Leishmania species, the determination of the specie is necessary to tackle the disease. On the other hand, the use of epidemiological and clinical findings is not sufficient to achieve this (1). Since the DNA of the parasite is specific to that organism similar to other living organisms, DNA can be used to identify and determine the species. Among the methods available for this purpose, using molecular methods particularly polymerase chain reaction (PCR) is useful (5). Leishmania needs to adapt to the ambient temperature and body temperature of mammalian hosts during its two-host life cycle. On this basis and according to recent studies, it has been proven that heat shock response and heat shock protein (HSP) play an important role in the process of adaptation and evolutionary changes of promastigotes in sandflies to Leishmania species isolated from mammalian hosts. HSP expression control in various eukaryotic systems has been widely studied and should be considered as an appropriate basis for comparison with regulatory mechanisms of Leishmania (6-8). Among various HSPs, HSP70 is better evolved and more conserved in both phases compared to other proteins of the family. It also has higher efficiency in biological processes within the cell than the rest of the proteins in this family. The HSP70 family of proteins is among the central components of many fundamental cellular processes in parasites including assembly of newly synthesized proteins in the cell, transferring proteins in the membrane, membrane processes of secretory proteins, proteolytic degradation of unstable proteins, controlling the activity of regulatory proteins and various immunological processes $(9,10$, and 11). In different species of Leishmania, HSP70I and HSP70II genes are similar in the $5^{\prime}$ untranslated region (5' UTR) and protein-coding region, but different in the $3^{\prime}$ UTR region $(6,12,13)$. Generally, these genes are arranged in a genetic cluster that includes 56 copies of the HSP70I followed by a copy of HSP70II. HSP70I's mRNA accumulates in response to heat shock treatment in various Leishmania species, which is later translated at 
both temperatures of 26 and $37{ }^{\circ} \mathrm{C}$. However, the mRNA of HSP70II does not accumulate in response to temperature; hence identification of the HSP70 gene, sequencing and genotyping in this region of the genome can be utilized for determination of species and differentiation of different Leishmania species $(6,12)$. Given the importance of this region in the parasite's genome and important biological processes of the parasite, this region can be targeted to control the intermediate host (sandflies) and develop human vaccines to combat the parasite in this area.

\section{MATERIAL AND METHODS}

This descriptive-analytical study was conducted in Chabahar and Mashhad during 2012-13. The samples were obtained from the Central Laboratory of Parasitology of Chabahar and Department of Parasitology, Emam Reza Hospital in Mashhad. Considering previous studies, the sample size of this study was considered 43 (33 L. major samples and $10 \mathrm{~L}$. tropica samples) and collected from the patients with suspected leishmaniasis. The samples were studied using direct and molecular methods. For direct examination, microscope slides were prepared and parasitic culture was performed on the Novy-MacNeal-Nicolle medium (NNN) and RPMI1640 media. The genomic DNA was extracted for molecular evaluation from the samples that were found as positive in the direct method. The parasite's DNA was extracted using genomic DNA extraction kit (Roche, Germany) according to the kit manufacturer's instructions. HSP1, HSP2, HSP3 and HSP4 specific primers with the following sequences were designed and sent to Takapoo Zist Co. for synthesis.
HSP1: 5'- TC GGA TCC GTG AAG GCG ACG AAC GGC - $3^{\prime}$

HSP2: 5'- AC AAG CTT GAA CAG CGC GTC GAT $-3^{\prime}$

HSP3: 5'- TC GGA TCC GTG TCC GCG GAG GAG - $3^{\prime}$

HSP4: 5'- TT AAG CTT GTT CTC CAG CTC CTT CTG $-3^{\prime}$

The extracted DNA was mixed with the primer in the three following forms:

1) HSP1 and HSP2 primers for amplification of NT- HSP70 region

2) HSP3 and HSP4 primers for amplification of CT- HSP70 region

3) HSP1 and HSP4 primers for amplification of the whole HSP70 fragment The PCR was carried out in a thermocycler according to the time-temperature program shown in Table 1. Electrophoresis of PCR products was performed on $0.7 \%$ agarose gel containing ethidium bromide and the obtained results were observed under a UV transilluminator. To interpret the size of the fragments, the positive and negative controls and the ladder markers of 50,100 and $500 \mathrm{bp}$ were used. Finally, PCR products were sent to the Takapoo Zist Co. for purification and sequencing.

\section{RESULTS}

In Chabahar, 24 of the 33 studied patients with suspected cutaneous leishmaniasis had positive smear and culture, while eight of the 10 studied patients in Mashhad were found as positive. Moreover, 11 negative samples were excluded from the study. Both HSP1 and HSP2 primers were used for the PCR to identify the NT-HSP70 region of $L$. major and L. tropica. After the PCR process, the bands with the size of $213 \mathrm{bp}$ were observed (Figure 1).

Table 1-The temperature and time programs given to the thermocycler

\begin{tabular}{|c|c|c|c|}
\hline $\begin{array}{c}\text { Number of } \\
\text { Cycles }\end{array}$ & Temperature & Time & Thermocycler Programs \\
\hline $\begin{array}{c}1 \\
35\end{array}$ & $\begin{array}{l}95^{\circ} \mathrm{C} \\
95^{\circ} \mathrm{C} \\
58^{\circ} \mathrm{C}\end{array}$ & $\begin{array}{l}5 \text { Minutes } \\
\text { 30 Seconds } \\
\text { 30 Seconds }\end{array}$ & $\begin{array}{c}\text { Initial Denaturation } \\
\text { Denaturation } \\
\text { Annealing }\end{array}$ \\
\hline 1 & $\begin{array}{l}72^{\circ} \mathrm{C} \\
72^{\circ} \mathrm{C}\end{array}$ & $\begin{array}{l}\text { One Minute } \\
10 \text { Minutes }\end{array}$ & $\begin{array}{c}\text { Extension } \\
\text { Final Extension }\end{array}$ \\
\hline
\end{tabular}


44/ Sequence Analysis of HSP70...

Figure 1- Gel electrophoresis of PCR products of the NT-HSP70 region and HSP70 fragment of L. majorin Chabahar

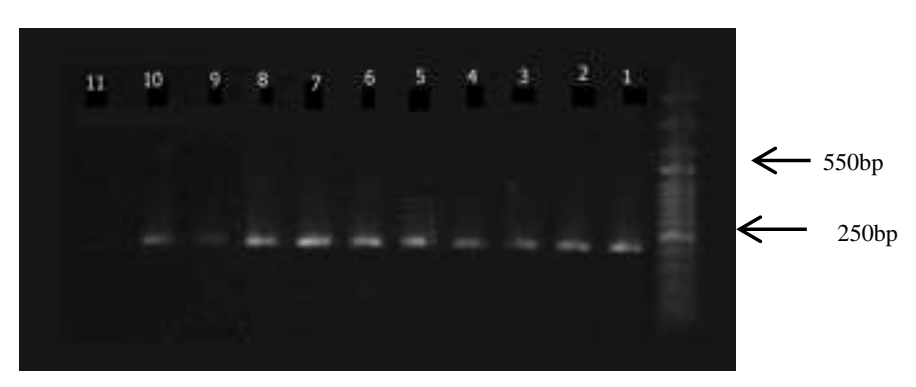

A

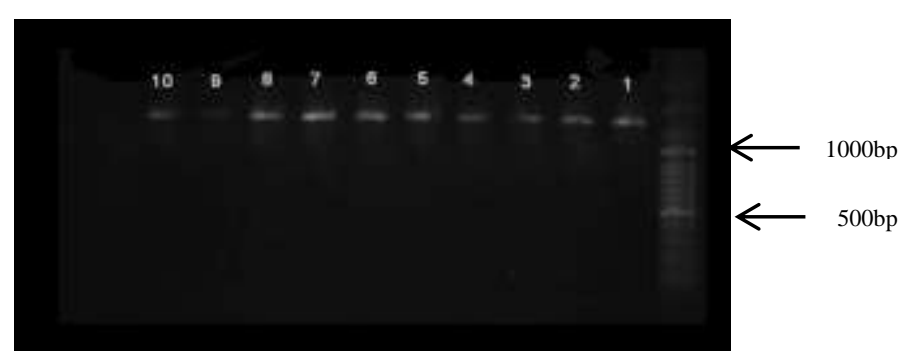

C

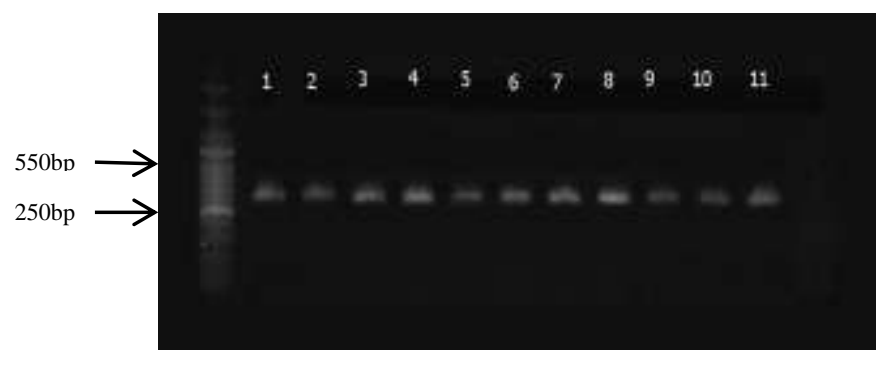

B

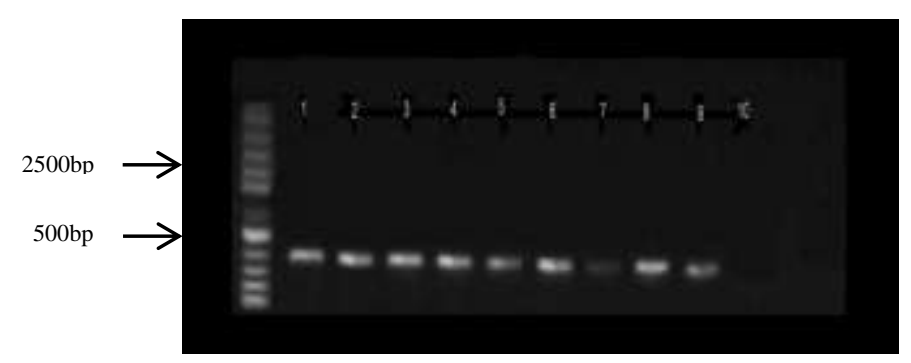

D

A: Right column: 50bp molecular marker, No.1: Standard L. major (MHOM / IR / 75 / ER), No.2-10: L. major from patients' samples (213bp), No.11: Negative control

B: Left column: 50 bp molecular marker, No.1: Standard L. major (MHOM / IR / 75 / ER), No.2: Standard L. tropica (MHOM / IR / 99 / YAZ1), No.3-11: L. major in patients' samples (342bp), No.12: Negative control

C: Right column: 100 bp molecular marker, No.1: Standard L. major (MHOM / IR / 75 / ER), No.2-10: L. major from patients' samples (1152bp), No.11: Negative control

D: Left column: 500bp molecular marker, No.1: Standard L. tropica (MHOM / IR / 99 / YAZ1), No.2-9: L. tropica from patients' samples (1962bp), No.10: Negative control

\section{DISCUSSION}

Of the 43 patients suspected of cutaneous leishmaniasis, $11(25 \%)$ had negative culture and smear. The sensitivity of parasite's presence in stained smears is affected by phase of the lesion. The diagnosis usually becomes difficult when the number of parasites is low. The factors such as heterogeneous distribution of amastigotes, sampling method and secondary bacterial contaminations can affect the result of direct smear. On the other hand, the superficial sampling of the lesions can minimize the possibility of finding the parasite in direct smear. After determining HSP70 gene sequence in all samples (L.major and L.tropica), the obtained sequences were compared with the findings of previous studies to determine the possible differences in the sequence of this gene among different Leishmania species. It was also determined whether there is a correlation between the HSP70 gene of L.major and $L$. tropica in the geographical area studied. For this purpose, the HSP70 gene sequence of other Leishmania species from other regions of the world, recorded in the GenBank, was extracted and compared with the obtained sequences in this study. The results of the HSP70 gene sequences from L.major in Chabahar and L.tropica in Mashhad were compared using the Basic Local Alignment Search Tool (BLAST) and 18 nucleotide differences were detected in the two tested Leishmania species. There were five nucleotide differences between 
L.major in Chabahar and L. donovani (IALE / CN / 83 / SELF-7 strain), reported by Zhano et al. (2012) in China. There were six nucleotide differences between L. major in Chabahar and L.aethiopica (MHOM / eT / 70 / L96 strain), reported by Van der Auwera et al. (2013) in Belgium. Finally, there were eight nucleotide differences between HSP70 of L. major in Chabahar and L. tropica (MHOM / KE / 81 / NLB_030B strain), reported by Fraga et al.(2010) in Cuba. The comparison of the nucleotide sequence of the HSP70 gene of $L$. major in Chabahar with other previously investigated species showed that the greatest nucleotide differences (258 nucleotides) are related to L.major (Friedlin strain), reported by Rogers et al. (2011) in the UK. Moreover, there were 100 nucleotide differences between $L$. tropica in Mashhad and L.braziliensis (MHOM / BR / 75 / M2904 strain), reported by Rogers et al. (2011) in the UK. There were 37 nucleotide differences between L. tropica in Mashhad and L.infantum clone Gpcm5, reported by Peacock et al. (2011) in the UK. Finally, there were 47 nucleotide differences between $L$. tropica in Mashhad and L. major (Friedlin strain), reported

\section{REFERENCES}

1. Vidyashankar C. Leishmania. eMedicine. 2006. Available at: URL: http://WWW.eMedicine.com/emerg/topic296.htm.Accesse d at Oct 11, 2006.

2. Mahmoodi MR, Tavakoli Afshar J, Mohajeri M, Fati AM,Yazdanpanah MJ, Shakeri MT, et al. Molecular Identification of Leishmania Species Causing Cutaneous Leishmaniasis in Mashhad, Iran. J Ilam Univ Med Sci. 2010; 18(2): 17-23. [In Persian].

3. Jamal khan Sh, Muneeb S. Cutaneous leishmaniasis in Pakestan. Dermatol online J. 2005 ؛ 11(1):4.

4. Azizi F, Hatami H, Janghorbani M. Epidemiology and Control of Common Disorders in Iran. Tehran: Eshtiagh Press. 2000.[Persian].

5. Farnoosh Gh. Identification of Leishmania species causing cutaneous leishmaniasis in Sabzevar (2007-2008). MSc Thesis, Mashhad: Mashhad University of Medical Sciences. 2008. [Persian].

6. Ramírez CA, Requena JM, Puerta CJ. Identification of the HSP70-II gene in Leishmania braziliensis HSP70 locus: genomic organization and UTRs characterization. Parasit Vectors. 2011; 4: 166. doi: 10.1186/1756-3305-4166.

7. Shapier M, Mceven JG, Jaff CL. Temperature on molecular processes which lead stage differentiation in leishmania. $\quad$ EMBO J. 1988; 7(9): 2895-2901. by Ivens et al. (2005) in the UK.

\section{CONCLUSION}

Due to the morphological similarities between Leishmania species and inability to differentiate species through parasitological methods, HSP70 gene can be used for identification of species, and prevention and treatment of disease. More thorough studies with larger sample size, longer study duration and a wider geographical area are recommended to utilize the obtain results in research on production of vaccines and pharmaceuticals against Leishmania strains in Iran.

\section{ACKNOWLEDGMENT}

This article was derived from the Master's thesis of the corresponding author. The authors would like to thank the Deputy of Research and Technology, Zabol University of Medical Sciences for financial support of this research project.

\section{CONFLICT OF INTEREST}

The authors declare no conflicts of interest regarding this manuscript.

8. Bente M, Harder S, Wiesgigl M. Developmentally induced change of the proteome in the protozoan parasite Leishmania donovani . Proteomics. 2003; 3(9): 1811-1829. 9. Hartl FU. Moleocular chaperones in cellular protien folding. Nature. 1996; 381(6583): 571-579.

10. Folgueira C, Requena JM. A post genomic view of the heat shock protiens in kintoplastids. FEMS Microbiol Rev. 2007; 31(4): 359-377.

11. Rafati S, Golami E, Hassani N, Ghaemmanesh F. Leishmania major heat protien (HSP70) is not protective in murine models of cutaneous leishmaniasis and stimulates strong humoral responses in cutaneous and visceral leishmaniasis patients. Vaccine. 2007; 25(21): 4159-69. PMID:17395340.

12. Fulgueira C, Quijada L, Soto M Abanades DR, Alonso C. The translational efficiencies of the two Leishmania infantum HSP70 mRNAs, differing in their 3`-untranslated region, are affected by shifts in the temperature of growth through different mechanism. J Biol Chem. 2005; 280(42): 35172-35183. PMID:16105831

13. Fulgueira C, Canavate C, Chicharo C, Requena JM. Genomic organisation and expression of the HSP70 locus in new and old world Leishmania species. Parasitilogy. 2007; 134(Pt3): 369-377. PMID:17054823 
